\title{
Balancing LTP and LTD
}

DOI:

10.1038/nrn2123
Two major forms of synaptic plasticity, long-term potentiation (LTP) and long-term depression (LTD) are cellular processes involved in learning and memory. Although they produce opposite effects on synaptic excitability, both LTP and LTD can occur at the same synapse in response to different patterns of activation of NMDA ( $N$-methyl-D-aspartate) receptors. This raises the question of whether specific mechanisms exist to prevent unwanted interference between the two processes. Collingridge and colleagues have now shown that LTP temporarily inhibits subsequent LTD induction at the same synapse and have uncovered a key role for glycogen synthase kinase $3 \beta$ (GSK3 $\beta$ ) in regulating the synaptic response downstream of NMDA receptor activation.

GSK3 $\beta$ has been implicated in neurological disorders such as Alzheimer's disease and mood disorders; however its roles in normal CNS functions have not yet been determined. In this study the authors showed that GSK $3 \beta$ is present in dendritic spines and is associated with AMPA ( $\alpha$-amino-3-hydroxy5-methyl-4-isoazolepropionic acid) receptors, the trafficking of which is believed to be important for changes in synaptic efficiency during LTP and LTD.

To examine a potential role for GSK3 $\beta$ in synaptic plasticity, the authors investigated the effects of inhibition of the enzyme on induction of LTD and LTP in rat hippocampal slices and revealed that GSK3 $\beta$ is essential for NMDA receptor-mediated LTD in this model. Inhibition of LTD by lithium was observed regardless of whether the inhibitor was applied before or after induction of LTD, suggesting that GSK $3 \beta$ might function in LTD maintenance. Furthermore, increased levels of GSK3 $\beta$ activity were associated with LTD induction. GSK3 $\beta$ activity is regulated by the phosphorylation state of a particular residue (Ser9), with dephosphorylation being associated with enhanced activity. Blocking the activity of protein phosphatase 1 reduced the dephosphorylation of Ser9 during LTD, indicating that this enzyme increases GSK3 $\beta$ activity during LTD.

The authors applied stimuli to cultured neurons that resulted in AMPA receptor insertion into the plasma membrane, mimicking events that take place during LTP induction. They showed that these stimuli also decreased AMPA receptor-associated GSK3 $\beta$ activity, suggesting that induction of LTP could inhibit GSK $3 \beta$ and therefore inhibit LTD. Indeed, the authors showed that the induction of LTP in hippocampal slices prevented the subsequent generation of LTD, an effect that lasted up to 1 hour after LTP induction. Blocking NMDA receptor activity abolished LTP-mediated inhibition of LTD, showing that transient activation of NMDA receptors mediates this effect. To investigate the downstream signalling pathways, the authors

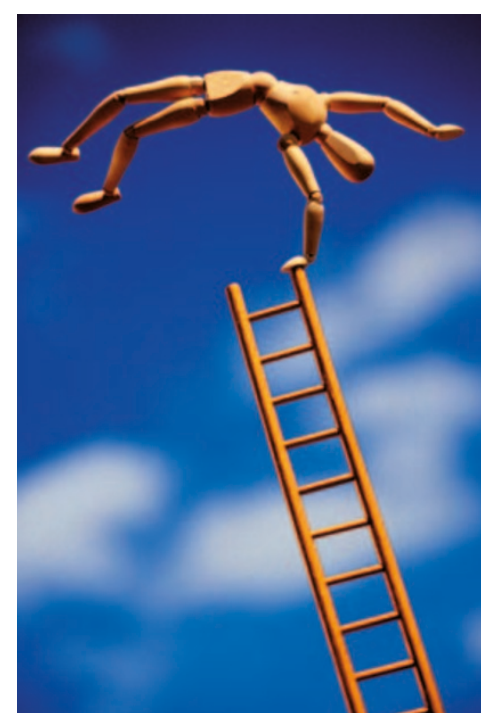

carried out a series of experiments using selective inhibitors of the phosphatidylinositol 3-kinase (PI3K)-Akt-GSK3 $\beta$ pathway and demonstrated that stimulation of this pathway, which leads to phosphorylation of GSK3 $\beta$ by Akt, is crucial for the prevention of LTD by LTP.

This study increases our understanding of the regulation of synaptic plasticity, showing one way in which prior synaptic activity can influence later synaptic changes. The benefits of such regulatory functions are unknown, but might include the temporary stabilization of synaptic modifications during a period in which the information is further consolidated within the brain. These findings also provide insights into the normal function of GSK $3 \beta$ in the CNS, which might help to uncover its roles in disease.

Katherine Whalley

ORIGINAL RESEARCH PAPER Peineau, S. et al. 